TITLE:

\title{
Photon creation in a resonant cavity with a nonstationary plasma mirror and its detection with Rydberg atoms
}

$\operatorname{AUTHOR}(S)$ :

Kawakubo, Toru; Yamamoto, Katsuji

\section{CITATION:}

Kawakubo, Toru ... [et al]. Photon creation in a resonant cavity with a nonstationary plasma mirror and its detection with Rydberg atoms. PHYSICAL REVIEW A 2011, 83(1): 013819.

ISSUE DATE:

2011-01-21

URL:

http://hdl.handle.net/2433/161771

RIGHT:

(C2011 American Physical Society 
PHYSICAL REVIEW A 83, 013819 (2011)

\title{
Photon creation in a resonant cavity with a nonstationary plasma mirror and its detection with Rydberg atoms
}

\author{
Toru Kawakubo and Katsuji Yamamoto \\ Department of Nuclear Engineering, Kyoto University, Kyoto 606-8501, Japan
}

(Received 9 November 2010; published 21 January 2011)

\begin{abstract}
We investigate the dynamical Casimir effect and its detection with Rydberg atoms. The photons are created in a resonant cavity with a plasma mirror of a semiconductor slab which is irradiated by periodic laser pulses. The canonical Hamiltonian is derived for the creation and annihilation operators, showing the explicit time variation in the couplings, which originates from the external configuration such as a nonstationary plasma mirror. The number of created photons is evaluated as squeezing from the Heisenberg equations with the Hamiltonian. Then the detection of the photons as the atomic excitations is examined through the atom-field interaction. Some consideration is made for a feasible experimental realization with a semiconductor plasma mirror.
\end{abstract}

DOI: 10.1103/PhysRevA.83.013819

PACS number(s): 42.50.Pq, 31.30.jh, 42.50.Lc, 42.50.Ct

\section{INTRODUCTION}

The quantum nature of the vacuum provides a variety of physically interesting phenomena, including the Casimir effect [1]. The so-called dynamical (nonstationary) Casimir effect (DCE), as well as the static force, has been investigated extensively (see [2-23], and references therein), where photons are created from the vacuum fluctuation because of a nonadiabatic change of the system such as vibration of a cavity or expansion of the universe. It is, however, difficult experimentally to realize the mechanical vibration of the cavity with a sufficient magnitude at the resonant frequency $\sim 1 \mathrm{GHz}$ which is required to create a significant number of photons for detection. As a feasible alternative, it has been proposed recently that the oscillating wall can be simulated by a plasma mirror of a semiconductor slab which is irradiated by periodic laser pulses [15] (see also $[16,17])$.

In this article, we investigate quantum mechanically the photon creation via the DCE and its detection with Rydberg atoms. We particularly intend to examine the experimental realization of DCE with a plasma mirror of a semiconductor slab [15,23]. In Sec. II, the canonical Hamiltonian for DCE is derived in terms of the creation and annihilation operators, where the field operators are expanded simply with the initial modes. Then, in Sec. III, the time-varying frequencies and squeezing couplings of the Hamiltonian are calculated in an effective $(1+1)$-dimensional scalar field model with a plasma mirror. They exhibit the enhancement of effective wall oscillation for the DCE which is simulated by the nonstationary plasma mirror. In Sec. IV, the number of photons created via the DCE is evaluated as squeezing from the Heisenberg equations for the creation and annihilation operators. The results appear to agree essentially with those obtained by the usual instantaneous-mode approach. In Sec. V, we investigate the excitation process of Rydberg atoms through the atom-field interaction, which is utilized to detect the created photons. Some conditions of the physical parameters are clarified for the efficient photon detection. In Sec. VI, the experimental realization of DCE with a semiconductor plasma mirror is discussed. Section VII is devoted to a summary.

\section{CANONICAL HAMILTONIAN}

We consider a scalar field in $3+1$ space-time dimensions as an effective description of the electromagnetic field in a resonant cavity. The Lagrangian is given by

$$
\mathcal{L}=\frac{1}{2} \epsilon(\mathbf{x}, t)(\dot{\phi})^{2}-\frac{1}{2}(\nabla \phi)^{2}-\frac{1}{2} m^{2}(\mathbf{x}, t) \phi^{2}
$$

( $\hbar=c=1)[7,9,10,18,19]$. Here $\epsilon(\mathbf{x}, t)$ and $m^{2}(\mathbf{x}, t)$ represent the dielectric permittivity and conductivity (effective mass term), respectively, in the matter region such as a semiconductor slab. As specified later, they are space-time dependent, simulating the boundary oscillation. Conventionally, the instantaneous modes $\bar{f}_{\alpha}(\mathbf{x}, t)$ (real, orthonormal, and complete) at each time $t$ with time-varying frequencies $\bar{\omega}_{\alpha}(t)$ are adopted according to the boundary oscillation:

$$
\left[-\nabla^{2}+m^{2}(\mathbf{x}, t)\right] \bar{f}_{\alpha}(\mathbf{x}, t)=\epsilon(\mathbf{x}, t) \bar{\omega}_{\alpha}^{2}(t) \bar{f}_{\alpha}(\mathbf{x}, t)
$$

with the orthonormalization

$$
\int_{V} \epsilon(\mathbf{x}, t) \bar{f}_{\alpha}(\mathbf{x}, t) \bar{f}_{\beta}(\mathbf{x}, t) d^{3} x=\delta_{\alpha \beta} /\left[2 \bar{\omega}_{\alpha}(t)\right] .
$$

Instead, we here specify the particle representation simply in terms of the initial modes:

$$
f_{\alpha}^{0}(\mathrm{x})=\bar{f}_{\alpha}(\mathbf{x}, t=0), \quad \omega_{\alpha}^{0}=\bar{\omega}_{\alpha}(t=0) .
$$

The canonical field operators in the Heisenberg picture are expanded with the creation and annihilation operators $a_{\alpha}^{\dagger}(t)$ and $a_{\alpha}(t)$ as

$$
\begin{gathered}
\phi(\mathbf{x}, t)=\sum_{\alpha}\left[a_{\alpha}(t)+a_{\alpha}^{\dagger}(t)\right] f_{\alpha}^{0}(\mathbf{x}), \\
\Pi(\mathbf{x}, t)=\epsilon(\mathbf{x}, 0) \sum_{\alpha} i \omega_{\alpha}^{0}\left[-a_{\alpha}(t)+a_{\alpha}^{\dagger}(t)\right] f_{\alpha}^{0}(\mathbf{x}),
\end{gathered}
$$


where $\Pi(\mathbf{x}, t)=\partial \mathcal{L} / \partial \dot{\phi}=\epsilon(\mathbf{x}, t) \dot{\phi}(\mathbf{x}, t)$. Then the canonical Hamiltonian is presented by the usual procedure as

$$
\begin{aligned}
H_{\mathrm{F}}(t)= & \int_{V} \frac{1}{2}\left\{\frac{\Pi^{2}}{\epsilon(\mathbf{x}, t)}+\phi\left[-\nabla^{2}+m^{2}(\mathbf{x}, t)\right] \phi\right\} d^{3} x \\
= & \sum_{\alpha} \omega_{\alpha}(t)\left(a_{\alpha}^{\dagger} a_{\alpha}+\frac{1}{2}\right)+\sum_{\alpha \neq \beta} \mu_{\alpha \beta}(t) a_{\alpha}^{\dagger} a_{\beta} \\
& +\sum_{\alpha, \beta} i\left[g_{\alpha \beta}(t) a_{\alpha}^{\dagger} a_{\beta}^{\dagger}-g_{\alpha \beta}^{*}(t) a_{\beta} a_{\alpha}\right],
\end{aligned}
$$

where the space integral is taken over the whole region $V$, which is fixed suitably (not time dependent) according to the physical setup, as illustrated later for the case of a cavity with a nonstationary plasma mirror. [The usual oscillating boundary may also be described as a periodic shift of the region of a high potential wall represented by $m^{2}(\mathbf{x}, t)$.] The explicit time dependence of the Hamiltonian $H_{\mathrm{F}}(t)$ in Eq. (7) represents the variation of the couplings which originates from the nonstationary behavior of the $c$ number external quantities $\epsilon(\mathbf{x}, t)$ and $m^{2}(\mathbf{x}, t)$. The second-order field equation (KleinGordon equation) is derived from the Heisenberg equations for $\phi(\mathbf{x}, t)$ and $\Pi(\mathbf{x}, t)$.

The mode frequencies $\omega_{\alpha}(t)$, intermode couplings $\mu_{\alpha \beta}(t)$, and squeezing terms $g_{\alpha \beta}(t)$ are calculated by considering the orthonormality of $f_{\alpha}^{0}(\mathbf{x})$, which obeys the wave equation with $\epsilon(\mathbf{x}, 0)$ and $m^{2}(\mathbf{x}, 0)$ :

$$
\begin{gathered}
\omega_{\alpha}(t)=\omega_{\alpha}^{0}+\mu_{\alpha \alpha}(t) \equiv \omega_{\alpha}^{0}+\delta \omega_{\alpha}(t), \\
\mu_{\alpha \beta}(t)=2 G_{\alpha \beta}^{\epsilon}(t)+2 G_{\alpha \beta}^{m}(t), \\
g_{\alpha \beta}(t)=-i\left[-G_{\alpha \beta}^{\epsilon}(t)+G_{\alpha \beta}^{m}(t)\right], \\
G_{\alpha \beta}^{\epsilon}(t)=\frac{1}{2} \omega_{\alpha}^{0} \omega_{\beta}^{0} \int_{\delta V(t)} \frac{\epsilon^{2}(\mathbf{x}, 0)}{\epsilon_{\Delta}(\mathbf{x}, t)} f_{\alpha}^{0}(\mathbf{x}) f_{\beta}^{0}(\mathbf{x}) d^{3} x, \\
G_{\alpha \beta}^{m}(t)=\frac{1}{2} \int_{\delta V(t)} m_{\Delta}^{2}(\mathbf{x}, t) f_{\alpha}^{0}(\mathbf{x}) f_{\beta}^{0}(\mathbf{x}) d^{3} x .
\end{gathered}
$$

The space integrals for $G_{\alpha \beta}^{\epsilon, m}(t)$ are actually evaluated in the subregion $\delta V(t)(\subseteq V)$, being possibly time dependent when a moving boundary is considered, where $\epsilon(\mathbf{x}, t)$ and $m^{2}(\mathbf{x}, t)$ vary in time as

$$
\begin{gathered}
\epsilon_{\Delta}^{-1}(\mathbf{x}, t) \equiv \epsilon^{-1}(\mathbf{x}, t)-\epsilon^{-1}(\mathbf{x}, 0), \\
m_{\Delta}^{2}(\mathbf{x}, t) \equiv m^{2}(\mathbf{x}, t)-m^{2}(\mathbf{x}, 0) .
\end{gathered}
$$

Here $G_{\alpha \beta}^{\epsilon, m}(0)=0$ with $\epsilon_{\Delta}^{-1}(\mathbf{x}, 0)=0$ and $m_{\Delta}^{2}(\mathbf{x}, 0)=0$ at $t=0$, as the Hamiltonian $H_{\mathrm{F}}(0)$ is diagonalized in terms of the initial modes $f_{\alpha}^{0}(\mathbf{x})$.

Similar formulas are presented for the effective Hamiltonian with the instantaneous modes [9,10]. This effective Hamiltonian involves even the time derivatives of the mode functions since the quantum time evolution is traced along the instantaneous modes. On the other hand, in the present approach, the time evolution is viewed on the initial modes according to the Heisenberg equations. The canonical Hamiltonian is calculated without the time derivatives of the mode functions and is readily applicable to various physical setups, for example, the case of a plasma mirror, clarifying its dependence on the experimental parameters. There may be some claim concerning the ambiguity in the particle representation and photon number since the basis modes are changing during the DCE. This ambiguity is, however, spurious physically (but might be essential for the case of the expanding universe, which is beyond the scope of this article). In fact, the instantaneous modes return to the initial modes at each period of the oscillation, where the photon number operators of the respective descriptions coincide with each other by definition. We can check explicitly that when the mode functions are not deformed largely in time, as usually considered, this canonical treatment provides essentially the same result for the DCE as the instantaneous-mode approach. The effects of the intermode couplings will be less significant in the instantaneous-mode approach, where the Hamiltonian is diagonalized at each time. Anyway, the intermode couplings are usually off resonant, providing subleading contributions to the DCE.

\section{VIBRATION WITH A PLASMA MIRROR}

We next calculate the time-varying frequencies and squeezing couplings of the Hamiltonian for DCE in an effective $(1+1)$-dimensional scalar field model with a nonstationary plasma mirror which is realized with a semiconductor slab irradiated by periodic laser pulses [15].

The dielectric response of the plasma is given by $\epsilon(\omega)=\epsilon_{1}\left[1-\left(\omega_{p}^{2} / \omega^{2}\right)\right]$, with the plasma frequency $\omega_{p}=$ $\left(n_{e} e^{2} / \epsilon_{1} m_{*}\right)^{1 / 2}$ in terms of the effective electron mass $m_{*}$ and the conduction electron number density $n_{e}$ proportional to the laser power $W_{\text {laser }} /$ pulse. This response for the dispersion relation, $\epsilon(\omega) \omega^{2}=\epsilon_{1} \omega^{2}-\left(n_{e} e^{2} / m_{*}\right)$, can be taken into account in the slab region $[l, l+\delta]$ around $x=l$ with a thickness $\delta(\ll L)$ as

$$
\epsilon(x, t)=\epsilon_{1}(t), \quad m^{2}(x, t)=m_{p}^{2}(t) \equiv n_{e}(t) e^{2} / m_{*},
$$

where $m_{p}^{2}(0)=0$ for $W_{\text {laser }}(0)=0$. (The spatial distribution of the conduction electrons along the $x$ direction may also be considered readily.) The instantaneous-mode functions are given as

$$
\bar{f}_{k}(x, t)= \begin{cases}D \sin k x & {[0, l),} \\ B e^{i k^{\prime} x}+C e^{-i k^{\prime} x} & {[l, l+\delta]: \text { slab }} \\ A \sin k[x-\delta+\xi(t)] & (l+\delta, L],\end{cases}
$$

with the dispersion relations

$$
\bar{\omega}_{k}^{2}=\left(k^{2}+\mathbf{k}_{\perp}^{2}\right) / \epsilon_{0}=\left(k^{\prime 2}+\mathbf{k}_{\perp}^{2}+m_{p}^{2}\right) / \epsilon_{1}
$$

$\left(k^{\prime}=i\left|k^{\prime}\right|\right.$ for $k^{\prime 2}<0$ with large $m_{p}^{2}$ ), where $\mathbf{k}_{\perp}$ is the momentum in the orthogonal spatial two dimensions (not shown explicitly) $[12,13,21]$. The Dirichlet boundary condition is adopted at $x=0, L$ with $\sin k[L-\delta+\xi(t)]=0$, corresponding to the case of transverse electric (TE) modes. The case of transverse magnetic (TM) modes can be treated in a similar way by adopting $m^{2}(x, t)=\left[\left(\partial n_{e} / \partial x\right) e^{2} /\left(\mathrm{k}_{\perp}^{2} m_{*}\right)\right]$ [23].

The diagonal couplings $\delta \omega_{k}(t)$ and $g_{k k}(t)$ are specifically calculated in Eqs. (8)-(12) with Eq. (16) for $f_{k}^{0}(x)$ at $t=0$ as

$$
\begin{gathered}
\delta \omega_{k}(t)=\omega_{k}^{0}\left[\delta_{\epsilon}(t)+\delta_{m}(t)\right] / L, \\
g_{k k}(t)=-(i / 2) \omega_{k}^{0}\left[-\delta_{\epsilon}(t)+\delta_{m}(t)\right] / L .
\end{gathered}
$$


Here the effective wall oscillation is enhanced as

$$
\begin{gathered}
\delta_{\epsilon}(t) / \delta \simeq-\left[\epsilon_{1}(0) / \epsilon_{0}\right]\left[1-\epsilon_{1}(0) / \epsilon_{1}(t)\right] \sin ^{2} k l, \\
\delta_{m}(t) / \delta \simeq\left[n_{e}(t) e^{2} / m_{*} \epsilon_{0}\left(\omega_{k}^{0}\right)^{2}\right] \sin ^{2} k l .
\end{gathered}
$$

This effect is almost proportional to the square of the mode function around the slab, $\left[f_{k}^{0}(l)\right]^{2} \propto \sin ^{2} k l$, since $\int_{l}^{l+\delta}\left[f_{k}^{0}(x)\right]^{2} d x \simeq\left[f_{k}^{0}(l)\right]^{2} \delta$ for $k^{\prime} \delta \sim\left[\epsilon_{1}(0) / \epsilon_{0}\right]^{1 / 2}(\delta / L) \ll$ 1 at $t=0$. If the slab is placed at the boundary $x=l=0$, $\sin ^{2} k l$ is replaced with $(k \delta)^{2} / 3 \sim(\delta / L)^{2} \ll 1$, as observed in Ref. [19], claiming that the DCE is suppressed in the TE mode. The significant photon creation, however, can take place even in the TE mode if the slab is placed apart from the boundaries $x=0, L$, which are the nodes of $f_{k}^{0}(x)$ $[18,23]$.

The shift $\xi(t)$ in the instantaneous mode of Eq. (16) is determined mainly proportional to $\delta$ to give the frequency modulation $\delta \bar{\omega}_{k}(t)$. The diagonal squeezing coupling $\bar{g}_{k k}(t)$ is then calculated with the formulas for the effective Hamiltonian $[9,10]$. After some calculations, we find the relations

$$
\delta \bar{\omega}_{k}(t) \simeq \delta \omega_{k}(t), \quad \bar{g}_{k k}(t) \simeq\left[i / 2 \bar{\omega}_{k}(t)\right] \dot{g}_{k k}(t),
$$

where the change of dielectric is assumed to be small, $\left|\epsilon_{1}(t)-\epsilon_{1}(0)\right| \ll \epsilon_{1}(0)$, as usual [19]. These relations in Eq. (22) ensure almost the same result for the DCE in the canonical and instantaneous-mode approaches (except for the small contribution of the off-resonant intermode couplings). This will be checked numerically in the next section.

The preceding calculations of $\delta \omega_{k}(t)$ and $g_{k k}(t)$ are valid up to $\left|\delta \omega_{k}(t)\right| / \omega_{k}^{0}=\left|\delta_{\epsilon}(t)+\delta_{m}(t)\right| / L \sim 0.1$, which is still a significant enhancement of the effective displacement $\left|\delta_{\epsilon, m}\right| \gg$ $\delta$ for the DCE. The present approach on the fixed basis, however, does not work effectively in an extreme situation where the mode functions are largely deformed in time with $\left|\delta \omega_{k}(t)\right| \sim \omega_{k}^{0}$. In such a case, the instantaneous-mode approach is rather suitable, though the deformation of the mode functions cannot be treated perturbatively [23]. Anyway, as seen in the following, a reasonable deformation to induce $\left|\delta \omega_{k}(t)\right| / \omega_{k}^{0} \sim 0.01-0.1$ is sufficient to create a significant number of photons for detection with atoms.

\section{PHOTON CREATION AS SQUEEZING}

Once the Hamiltonian is presented in terms of the creation and annihilation operators, the time evolution for the DCE is determined by the Heisenberg equations $\dot{a}_{\alpha}(t)=$ $i\left[H_{\mathrm{F}}(t), a_{\alpha}(t)\right]$ and $\dot{a}_{\alpha}^{\dagger}(t)=i\left[H_{\mathrm{F}}(t), a_{\alpha}^{\dagger}(t)\right]$. It is described as the Bogoliubov transformation:

$$
\begin{aligned}
& a_{\alpha}(t)=A_{\alpha \beta}(t) a_{\beta}+B_{\alpha \beta}^{*}(t) a_{\beta}^{\dagger}, \\
& a_{\alpha}^{\dagger}(t)=A_{\alpha \beta}^{*}(t) a_{\beta}^{\dagger}+B_{\alpha \beta}(t) a_{\beta} .
\end{aligned}
$$

The master equations for the Bogoliubov transformation are derived from the Heisenberg equations as

$$
\begin{gathered}
\dot{A}_{\alpha \beta}=-i \omega_{\alpha}(t) A_{\alpha \beta}-i \mu_{\alpha \gamma}(t) A_{\gamma \beta}+2 g_{\alpha \gamma} B_{\gamma \beta}, \\
\dot{B}_{\alpha \beta}=i \omega_{\alpha}(t) B_{\alpha \beta}+i \mu_{\alpha \gamma}^{*}(t) B_{\gamma \beta}+2 g_{\alpha \gamma}^{*} A_{\gamma \beta},
\end{gathered}
$$

where the intermode couplings are renamed suitably as $\mu_{\alpha \gamma}\left(1-\delta_{\alpha \gamma}\right) \rightarrow \mu_{\alpha \gamma}$ with $\mu_{\alpha \alpha} \equiv 0$.

In the following, we illustrate the characteristic features of DCE by concentrating on a single resonant mode with timevarying frequency $\omega(t)=\omega_{0}+\delta \omega(t)$ and squeezing coupling $g(t)$ (the mode index $k$ omitted). The intermode couplings will not provide significant contributions since they are fairly off resonant generally for the nonequidistant frequency differences $[11,13,21]$. The master equations read

$$
\dot{A}=-i \omega(t) A+2 g(t) B, \quad \dot{B}=i \omega(t) B+2 g^{*}(t) A
$$

for the Bogoliubov transformation:

$$
a(t)=A(t) a+B^{*}(t) a^{\dagger}, \quad a^{\dagger}(t)=A^{*}(t) a^{\dagger}+B(t) a .
$$

The solution is expressed as squeezing and phase rotation [2]:

$$
A(t)=\cosh r(t) e^{i \phi_{A}(t)}, \quad B(t)=\sinh r(t) e^{i \phi_{B}(t)},
$$

with the initial condition $A(0)=1, B(0)=0$, ensuring $|A(t)|^{2}-|B(t)|^{2}=1$.

An analytic solution for $A(t)$ and $B(t)$ is obtained in the rotating-wave approximation (RWA) by replacing

$$
\begin{array}{ll}
\omega(t) \rightarrow \omega_{0}+\langle\delta \omega\rangle & (\text { average }), \\
g(t) \rightarrow\langle g\rangle_{\Omega} e^{-i \Omega t} & \text { (Fourier component), }
\end{array}
$$

where $\omega_{0}=\omega(0)$. By noting the time evolution of the number operator $a^{\dagger}(t) a(t)=|B(t)|^{2} a a^{\dagger}+\ldots$, we obtain the photon creation via DCE (vacuum squeezing) as

$$
\begin{aligned}
n_{\gamma}(t) & =\left\langle 0\left|a^{\dagger}(t) a(t)\right| 0\right\rangle=|B(t)|^{2} \\
& \simeq\left|\frac{2\langle g\rangle_{\Omega}}{\chi}\right|^{2} \times \begin{cases}\sinh ^{2} \chi t & \left(|\Delta|<\left|2\langle g\rangle_{\Omega}\right|\right), \\
|\chi|^{2} t^{2} & \left(|\Delta|=\left|2\langle g\rangle_{\Omega}\right|\right), \\
\sin ^{2}|\chi| t & \left(|\Delta|>\left|2\langle g\rangle_{\Omega}\right|\right),\end{cases}
\end{aligned}
$$

with the effective squeezing rate

$$
\chi=\sqrt{\left|2\langle g\rangle_{\Omega}\right|^{2}-\Delta^{2}} .
$$

Here the detuning $\Delta$ is introduced for the frequency $\Omega$ of laser pulses $[12,13]$ as

$$
\Omega=2\left(\omega_{0}+\langle\delta \omega\rangle+\Delta\right) .
$$

The resonance condition for DCE is then given by

$$
\Omega(\text { resonance })=2\left(\omega_{0}+\langle\delta \omega\rangle\right),
$$

involving the average shift of the frequency $\langle\delta \omega\rangle[18,23]$ rather than the naive condition $\Omega=2 \omega_{0}$. If $\Omega=2 \omega_{0}$ is taken with $\Delta=-\langle\delta \omega\rangle$, the squeezing rate $\chi$ is significantly reduced, even possibly becoming imaginary with $n_{\gamma}(t) \lesssim 1$ oscillating as $\sin ^{2}|\chi| t$. The photon damping with the factor $e^{-\Gamma t}$ because of the cavity loss should further be taken into account, where

$$
\Gamma=\omega_{0} / Q,
$$

with the cavity quality factor $Q$. Hence the threshold condition for the squeezing by DCE is placed as

$$
\chi>\Gamma / 2,
$$

which is readily satisfied with a large enough $Q$. 


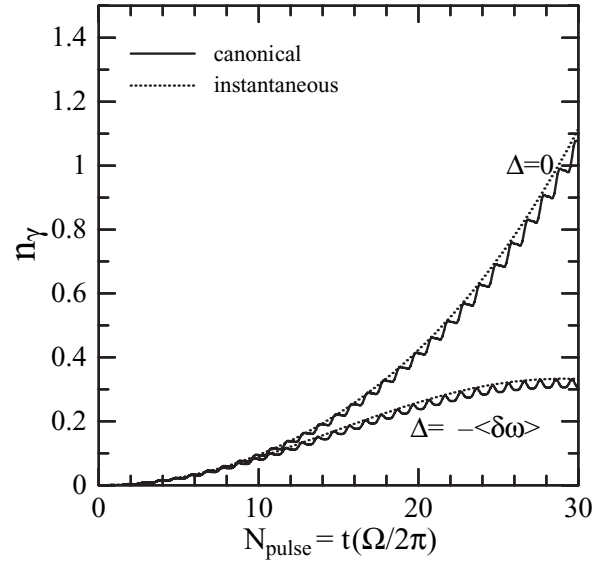

FIG. 1. Photon creation $n_{\gamma}(t)$ (linear plot) in the early stage of DCE for $N_{\text {pulse }}=t(\Omega / 2 \pi) \leqslant 30$ (the number of periodic laser pulses). The results of the canonical and instantaneous-mode approaches are shown with the solid and dotted curves, respectively. The parameters are taken typically as $\langle\delta \omega\rangle=0.02 \omega_{0}, 2\langle g\rangle_{\Omega}=i 0.01 \omega_{0}$, and $\Delta=0$ (on-resonance, upper curves) and $-\langle\delta \omega\rangle$ (off-resonance, lower curves) for $\Omega$.

We have solved numerically the master equations in Eq. (27) without the RWA. The time-varying couplings are taken typically as $\omega(t)=\omega_{0}+\langle\delta \omega\rangle(1-\cos \Omega t)$ and $g(t)=2\langle g\rangle_{\Omega}(1-\cos \Omega t)$, where $\left|2\langle g\rangle_{\Omega}\right| \sim|\langle\delta \omega\rangle| / 2$, as indicated in Eqs. (18) and (19) for the plasma mirror. The instantaneous-mode solution has also been obtained by considering the relations $\delta \bar{\omega}(t)=\delta \omega(t)$ and $\bar{g}(t)=[i / 2 \bar{\omega}(t)] \dot{g}(t)$ in Eq. (22). In Fig. 1, the photon creation $n_{\gamma}(t)$ in the early stage of DCE is plotted for $N_{\text {pulse }}=t(\Omega / 2 \pi) \leqslant 30$ (the number of periodic laser pulses). The results of the canonical and instantaneous-mode approaches are shown with the solid and dotted curves, respectively. Here the parameters are taken typically as $\langle\delta \omega\rangle=0.02 \omega_{0}, 2\langle g\rangle_{\Omega}=i 0.01 \omega_{0}$, and $\Delta=0$

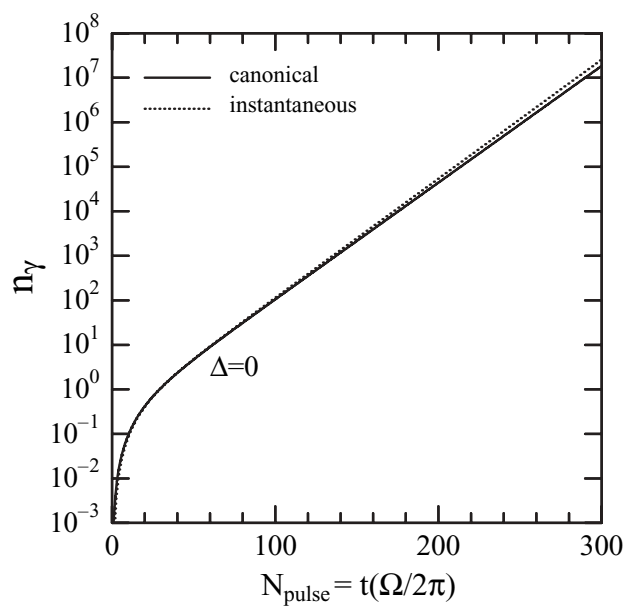

FIG. 2. Photon creation $n_{\gamma}(t)$ (log plot) through the DCE period for $N_{\text {pulse }}=t(\Omega / 2 \pi) \leqslant 300$. The results of the canonical and instantaneous-mode approaches are shown with the solid and dotted curves, respectively. The parameters are taken typically as $\langle\delta \omega\rangle=$ $0.02 \omega_{0}, 2\langle g\rangle_{\Omega}=i 0.01 \omega_{0}$, and $\Omega=2.04 \omega_{0}(\Delta=0)$. (upper curves) and $-\langle\delta \omega\rangle$ (lower curves) for $\Omega$ in Eq. (34). We can see that $n_{\gamma}(t)$ increases rapidly via the DCE on the resonance with $\Omega=2\left(\omega_{0}+\langle\delta \omega\rangle\right)(\Delta=0)$, while $n_{\gamma}(t)$ does not grow for $\Omega=2 \omega_{0}(\Delta=-\langle\delta \omega\rangle)$ because of the effective detuning brought by the average shift $\langle\delta \omega\rangle$. In Fig. 2, the photon creation $n_{\gamma}(t)$ is plotted through the DCE period for $N_{\text {pulse }}=t(\Omega / 2 \pi) \leqslant 300$. The squeezing rate is determined from this plot to be $\chi \simeq 0.01 \omega_{0}$, as indicated in Eq. (33) with $\Delta=0$. This result confirms that a large number of photons can be created via the DCE with a reasonable squeezing rate $\chi \sim 0.01 \omega_{0}$ when the laser pulses are applied many times. It is also found that the canonical and instantaneous-mode approaches provide almost the same result (except for the small contribution of the off-resonant intermode couplings). The analytic solution under the RWA in Eq. (32) overlaps almost with the instantaneous-mode result, though it is not plotted explicitly in Figs. 1 and 2.

We briefly discuss the effect of the intermode couplings. Specifically, the coupling $\mu_{12} a_{1}^{\dagger} a_{2}+\mu_{12}^{*} a_{2}^{\dagger} a_{1}$ between the modes 1 and 2 becomes resonant under a condition $\omega_{2}^{0}=$ $3 \omega_{1}^{0} \rightarrow \omega_{2}^{0}-\omega_{1}^{0}=2 \omega_{1}^{0} \approx \Omega$ for the case of the $\mathrm{TE}_{111}$ and $\mathrm{TE}_{115}$ modes in a cubic cavity because of the relation $\left(1^{2}+\right.$ $\left.1^{2}+5^{2}\right)^{1 / 2}=3\left(1^{2}+1^{2}+1^{2}\right)^{1 / 2}$. Then, through this resonant intermode coupling, the significant photon creation occurs in both modes 1 and 2 as $n_{\gamma_{1}}(t) \sim n_{\gamma_{2}}(t)$, increasing the total of photon numbers $[13,21,23]$. The photons of mode 2 are, however, fairly off resonant with the Rydberg atoms tuned to detect the photons of mode 1 . Hence they cannot be detected efficiently.

\section{DETECTION WITH RYDBERG ATOMS}

The photons created via the DCE are detected suitably by Rydberg atoms with principal quantum number $n \approx 100$ and transition frequency $\sim 1 \mathrm{GHz}[11,23]$. Rydberg atoms may be treated as a two-level system with a transition frequency $\omega_{e}$ for the resonant photon absorption with $\omega_{e} \approx \omega_{0}$. They are initially prepared in the lower level $|g\rangle$ and injected into the cavity. A part of these atoms are excited to the upper level $|e\rangle$ by absorbing the photons and are detected outside the cavity as the signal of photons. Recently, a high-sensitivity measurement of blackbody radiation has been performed at a frequency $2.527 \mathrm{GHz}$ and low temperatures $67 \mathrm{mK}-1 \mathrm{~K}$ by employing a Rydberg-atom cavity detector with a newly developed selective field ionization scheme for $n \approx 100$ (the atoms excited by absorbing photons are selectively ionized by applying an electric field) [24]. Here we note that in order to observe purely the vacuum squeezing via DCE, the cavity should be cooled well below $100 \mathrm{mK}$ to suppress the thermal photons as $n_{\gamma}$ (thermal) $\ll 1$. In fact, if photons are present initially with an expectation value $\left\langle a^{\dagger} a\right\rangle$, they are also amplified by the DCE as $\left(1+2|B(t)|^{2}\right)\left\langle a^{\dagger} a\right\rangle$.

Consider that $N_{\text {Ryd }}$ Rydberg atoms (actually, $N_{\text {Ryd }} \sim 100$ 1000 [24]), which are all prepared at the lower level $|g\rangle$, are injected into the cavity to detect the created photons after the period of DCE, for simplicity of argument. (The following features for the photon detection are essentially valid even if the atomic beam is injected continuously during and after the DCE, as discussed later.) The $n_{\gamma}$ photons and $N_{\text {Ryd }}$ atoms (all 
located at the same position, for simplicity) are coupled with the Jaynes-Cummings Hamiltonian under the RWA as

$$
H_{\mathrm{AF}}=\kappa \sqrt{N_{\mathrm{Ryd}}}\left(a D_{+}+a^{\dagger} D_{-}\right) .
$$

(The effect of the counterrotating terms is negligible near the resonance.) Here the collective atomic spinlike operators are defined (in the Schrödinger picture) [25] by

$$
\begin{aligned}
& D_{+} \equiv \sum_{i=1}^{N_{\text {Ryd }}}|e\rangle\left\langle\left. g\right|_{(i)} / \sqrt{N_{\text {Ryd }}},\right. \\
& D_{-} \equiv \sum_{i=1}^{N_{\text {Ryd }}}|g\rangle\left\langle\left. e\right|_{(i)} / \sqrt{N_{\text {Ryd }}},\right.
\end{aligned}
$$

and the complex phase for $\kappa$ is absorbed in the atomic levels. The single atom-photon coupling $\kappa$ is explicitly given by

$$
\kappa=d \sqrt{\omega_{0} / 2 \epsilon_{0} V}\left[\left|f^{0}\left(\mathbf{x}_{1}\right)\right| /\left|f^{0}\left(\mathbf{x}_{0}\right)\right|\right]
$$

in terms of the magnitude of the electric dipole transition matrix element $d$, the cavity volume $V$, and the mode function $f^{0}(\mathbf{x})$, where $\mathbf{x}_{1}$ and $\mathbf{x}_{0}$ represent the atomic position and the antinode, respectively. The collective atom-photon coupling is suitably defined by

$$
\bar{\kappa}=\kappa \sqrt{N_{\mathrm{Ryd}}} .
$$

The single atom-field coupling is typically $\kappa \sim 3 \times 10^{3} \mathrm{~s}^{-1}$ at the antinode for the Rydberg atom of principal quantum number $n \approx 100$ with $\omega_{e} \approx \omega_{0} \sim 1.5 \times 10^{10} \mathrm{~s}^{-1}(2.4 \mathrm{GHz} \times$ $2 \pi)$ and $V \sim(0.1 \mathrm{~m})^{3}[24,25]$. Then the collective coupling amounts to $\bar{\kappa} \sim 10^{5} \mathrm{~s}^{-1} \sim 10^{-5} \omega_{0}$ for $N_{\mathrm{Ryd}} \sim 10^{3}$, which is still much smaller than the resonant frequency $\omega_{e} \approx \omega_{0}$.

The commutation relations among the collective operators are given by

$$
\begin{aligned}
& {\left[D_{+}, D_{-}\right] }=D_{z} \\
& \equiv \sum_{i=1}^{N_{\text {Ryd }}}\left[|e\rangle\left\langle\left. e\right|_{(i)}-\mid g\right\rangle\left\langle\left. g\right|_{(i)}\right] / N_{\text {Ryd }},\right. \\
& {\left[D_{z}, D_{ \pm}\right]= \pm\left(2 / N_{\text {Ryd }}\right) D_{ \pm} . }
\end{aligned}
$$

The operators $\hat{N}_{e}$ and $\hat{N}_{g}$, to represent the populations of the upper and lower levels $|e\rangle$ and $|g\rangle$, respectively, are given by

$$
\begin{gathered}
\hat{N}_{e}=\sum_{i=1}^{N_{\text {Ryd }}}|e\rangle\left\langle\left. e\right|_{(i)}=\left(N_{\text {Ryd }} / 2\right)\left(1+D_{z}\right),\right. \\
\hat{N}_{g}=\sum_{i=1}^{N_{\text {Ryd }}}|g\rangle\left\langle\left. g\right|_{(i)}=\left(N_{\text {Ryd }} / 2\right)\left(1-D_{z}\right),\right.
\end{gathered}
$$

satisfying the completeness

$$
\hat{N}_{e}+\hat{N}_{g}=\sum_{i=1}^{N_{\mathrm{Ryd}}}\left[|e\rangle\left\langle\left. e\right|_{(i)}+\mid g\right\rangle\left\langle\left. g\right|_{(i)}\right] \equiv N_{\mathrm{Ryd}} .\right.
$$

The created photons are detected by counting the number of excited atoms, which is represented by $\hat{N}_{e}$ with eigenvalues $0,1, \ldots, N_{\text {Ryd }}$. The initial atomic state is prepared as

$$
\left|0_{e}\right\rangle=\left|g_{1}, g_{2}, \ldots, g_{N_{\mathrm{Ryd}}}\right\rangle,
$$

which is an eigenstate of $\hat{N}_{e}$ with zero atomic excitation satisfying $D_{-}\left|0_{e}\right\rangle=0$. The one-excitation state is generated as

$$
\begin{aligned}
\left|1_{e}\right\rangle & =D_{+}\left|0_{e}\right\rangle \\
& =\frac{1}{\sqrt{N_{\mathrm{Ryd}}}} \sum_{i=1}^{N_{\mathrm{Ryd}}}\left|g_{1}, \ldots, e_{i}, g_{i+1}, \ldots, g_{N_{\mathrm{Ryd}}}\right\rangle,
\end{aligned}
$$

and so on for the multiexcitation states.

The Heisenberg equations are derived by taking the total Hamiltonian $H_{\mathrm{A}}+H_{\mathrm{AF}}+H_{\mathrm{F}}$ with $H_{\mathrm{A}}=\left(N_{\mathrm{Ryd}} / 2\right) \omega_{e} D_{z}$ for the free atomic system:

$$
\begin{gathered}
\dot{a}=-i \omega_{0} a-i \bar{\kappa} D_{-}, \\
\dot{D}_{-}=-i \omega_{e} D_{-}+i \bar{\kappa} a D_{z}, \\
\dot{D}_{z}=-i\left(2 / N_{\mathrm{Ryd}}\right) \bar{\kappa}\left(a D_{+}-a^{\dagger} D_{-}\right) .
\end{gathered}
$$

We solve these equations perturbatively to see the evolution of the atomic excitation $N_{e}(t)=\left\langle\hat{N}_{e}(t)\right\rangle$. First, Eqs. (50) and (51) for $a(t)$ and $D_{-}(t)=D_{+}^{\dagger}(t)$ are integrated up to the first order of $\bar{\kappa}$ with the initial atomic operators $D_{ \pm}\left(t_{1}\right)$ in Eqs. (39) and (40) and the photon operator $a\left(t_{1}\right)$ at $t=t_{1}$ after the DCE with one sequence of $N_{\text {pulse }}$ laser pulses for the duration

$$
t_{1}=N_{\text {pulse }}(2 \pi / \Omega) \text {. }
$$

Then the results are applied to Eq. (52) to obtain $D_{z}(t)$ up to the second order of $\bar{\kappa}$. This determines the atomic excitation as

$$
\begin{aligned}
N_{e}(t) & =\left\langle\hat{N}_{e}(t)\right\rangle=\left(N_{\mathrm{Ryd}} / 2\right)\left[1+\left\langle D_{z}(t)\right\rangle\right] \\
& \simeq n_{\gamma}\left(2 \bar{\kappa} / \Delta_{e}\right)^{2} \sin ^{2}\left[\Delta_{e}\left(t-t_{1}\right) / 2\right],
\end{aligned}
$$

where the atomic detuning is given by

$$
\Delta_{e}=\omega_{e}-\omega_{0} .
$$

In these calculations, the following relations are considered: $\left\{a, a^{\dagger}\right\} D_{z}+\left\{D_{+}, D_{-}\right\}=2\left(a^{\dagger} a D_{z}+D_{+} D_{-}\right),\left[a, a^{\dagger}\right] D_{z}-$ $\left[D_{+}, D_{-}\right]=0,\left\langle 0_{e}\left|D_{ \pm}\left(t_{1}\right)\right| 0_{e}\right\rangle=0,\left\langle 0_{e}\left|D_{+}\left(t_{1}\right) D_{-}\left(t_{1}\right)\right| 0_{e}\right\rangle=$ $0,\left\langle 0_{e}\left|D_{z}\left(t_{1}\right)\right| 0_{e}\right\rangle=-1$, and $\left\langle 0\left|a^{\dagger}\left(t_{1}\right) a\left(t_{1}\right)\right| 0\right\rangle=n_{\gamma}$ (the photons created via the DCE). Note here that $N_{e}(t) \ll N_{\text {Ryd }}$ with $\left\langle D_{z}\right\rangle \approx-1$ in the early epoch of photon detection (the linear regime). Although it is difficult in practice to trace exactly the time evolution beyond the linear regime for the system of the many atoms interacting with the resonant cavity mode, we may survey the essential features for the atomic excitation to detect the photons as follows.

Suppose that $n_{\gamma} \gg N_{\text {Ryd }}$, namely, the photons are created much more than the Rydberg atoms, as desired and feasible experimentally. Then the atomic excitation is eventually saturated as $N_{e}(t) \sim(\bar{\kappa} t)^{2} n_{\gamma} \sim N_{\mathrm{Ryd}}$ for $t \sim 1 /\left(\kappa \sqrt{n_{\gamma}}\right)$, which is expected by extrapolating Eq. (54) roughly up to $\bar{\kappa} t \sim \sqrt{N_{\mathrm{Ryd}} / n_{\gamma}} \ll 1$ near the resonance $\Delta_{e} \approx 0$ (henceforth $t-t_{1} \rightarrow t$ ). This excitation process may be viewed as the onset of Rabi oscillation between $|g\rangle$ and $|e\rangle$ at a rate

$$
\Omega_{e} \sim \kappa \sqrt{n_{\gamma}},
$$

which takes place almost independently for the $N_{\text {Ryd }}$ atoms in the presence of the large field (many photons with $n_{\gamma} \gg N_{\text {Ryd }}$ ). 
On the other hand, if $n_{\gamma}<N_{\text {Ryd }}$, though less interesting experimentally, the excitation is exchanged between the atoms and field as $N_{e}(t) \sim n_{\gamma} / 2$ on average for $\bar{\kappa} t \sim 1$. This may be understood from the fact that the interaction Hamiltonian $H_{\mathrm{AF}}$ in Eq. (38) describes the oscillation with a rate $\Omega_{e} \sim \bar{\kappa}=\kappa \sqrt{N_{\mathrm{Ryd}}}$ between the atomic and field operators in the linear regime. The collective atomic excitation can be treated as a quantum oscillator, satisfying approximately the bosonic commutation relation $\left[D_{-}, D_{+}\right] \approx-\left\langle D_{z}\right\rangle \approx 1$ with $n_{\gamma} \ll N_{\text {Ryd }}$ in Eq. (43), that is, $D_{+}$and $D_{-}$act as the creation and annihilation operators, respectively [25].

The cavity loss eventually becomes significant for $t \gtrsim 1 / \Gamma$. Then the atomic excitation is also relaxed with a rate

$$
\Gamma_{e} \sim \begin{cases}4(\bar{\kappa} / \Gamma)^{2} \Gamma & (\bar{\kappa}<\Gamma / 4) \\ \Gamma / 2 & (\bar{\kappa} \geqslant \Gamma / 4)\end{cases}
$$

through the transition $|e\rangle \rightarrow|g\rangle+\gamma$ and the loss of the emitted photon in the cavity [25]. We also note that the atomfield interaction terminates when the atoms transit through the cavity. The atomic transit time is given by

$$
t_{\mathrm{tr}}=L / v \equiv \Gamma_{\mathrm{tr}}^{-1},
$$

where $v$ and $L$ are the atomic velocity and the cavity length, respectively. We have typically

$$
\Gamma_{\mathrm{tr}} \sim \frac{300 \mathrm{~m} / \mathrm{s}}{0.1 \mathrm{~m}}=3 \times 10^{3} \mathrm{~s}^{-1},
$$

which is comparable to the single atom-field coupling $\kappa$. By considering these damping effects, we realize that the created photons are detected efficiently with the atoms under the conditions

$$
\begin{gathered}
\Omega_{e} \gtrsim \Gamma, \Gamma_{\mathrm{tr}}, \\
\Gamma_{\mathrm{tr}} \gtrsim \Gamma_{e} .
\end{gathered}
$$

The atomic excitation should take place for $t \sim \Omega_{e}^{-1}$ before the significant loss of the created photons because of the cavity damping $\left(\Gamma \geqslant 2 \Gamma_{e}\right)$ and the actual cutoff of the atom-field interaction by the atomic transit $\left(\Gamma_{\mathrm{tr}}\right)$. It is also required that the excitation damping $\left(\Gamma_{e}\right)$ induced by the cavity loss does not become significant before the atoms transit through the cavity $\left(\Gamma_{\text {tr }}\right)$.

As investigated so far, if the photons are created copiously via the DCE with $n_{\gamma} \gg N_{\text {Ryd }}$, they are detected by the atomic excitation as

$$
N_{e}\left(t_{\mathrm{tr}}\right) \sim N_{\mathrm{Ryd}} / 2
$$

Here the condition $\Omega_{e} \gtrsim \Gamma_{\text {tr }}$ is less restrictive, requiring merely $n_{\gamma} \gtrsim\left(\Gamma_{\mathrm{tr}} / \kappa\right)^{2} \sim 1$ for $\Gamma_{\mathrm{tr}} \sim \kappa$. The atomic detuning may be suppressed readily as $\Delta_{e}<\Omega_{e}$, for example, for $\Omega_{e} \sim 3 \times$ $10^{6} \mathrm{~s}^{-1}$ with $\kappa \sim 3 \times 10^{3} \mathrm{~s}^{-1}$ and $n_{\gamma} \sim 10^{6}$. The conditions $\Omega_{e} \gtrsim \Gamma$ and $\Gamma_{\operatorname{tr}} \gtrsim \Gamma_{e} \sim 2(\bar{\kappa} / \Gamma)^{2} \Gamma(\bar{\kappa}<\Gamma / 4)$ imply lower and upper bounds, respectively, on the cavity quality factor:

$$
\left(\omega_{0} / \kappa\right) / \sqrt{n_{\gamma}} \lesssim Q \lesssim\left(\omega_{0} / \kappa\right)\left(\Gamma_{\text {tr }} / \kappa\right) / N_{\mathrm{Ryd}},
$$

where $\omega_{0} / \kappa \sim 5 \times 10^{6}$. These bounds are combined as a requirement for the number of created photons:

$$
n_{\gamma} \gtrsim\left(\kappa / \Gamma_{\text {tr }}\right)^{2} N_{\text {Ryd }}^{2} \gg N_{\text {Ryd }}
$$

For example, we estimate $Q \sim 5 \times 10^{3}$ and $n_{\gamma} \sim 10^{6}$ for $\Gamma_{\operatorname{tr}} \sim$ $\kappa$ and $N_{\text {Ryd }} \sim 10^{3}$. This range of $Q$ meets consistently the condition $\bar{\kappa}<\Gamma / 4$ for $\Gamma_{e}$.

On the other hand, if $\Gamma_{e}=\Gamma / 2(\bar{\kappa} \geqslant \Gamma / 4)$, the condition $\Gamma_{\mathrm{tr}} \gtrsim \Gamma_{e}$ places a significant bound:

$$
Q \gtrsim \omega_{0} / \Gamma_{\mathrm{tr}} \sim 5 \times 10^{6} .
$$

This range of $Q$ meets consistently the condition $\bar{\kappa} \geqslant \Gamma / 4$ for $\Gamma_{e}$. We also note that $N_{e}\left(t_{\mathrm{tr}}\right) \sim n_{\gamma} / 2$ for $n_{\gamma}<N_{\text {Ryd }}$. In this case, with $\Omega_{e} \sim \bar{\kappa}$, the condition $\Omega_{e} \gtrsim \Gamma$ implies $\bar{\kappa} \geqslant \Gamma / 4$. Hence the preceding range of $Q$ in Eq. (65) is effective either for $n_{\gamma} \gtrsim N_{\text {Ryd }}$ or $n_{\gamma}<N_{\text {Ryd }}$.

The atomic beam may be injected continuously through the period of DCE. Then we can show that the atomic excitation is squeezed together as $N_{e}(t) \sim\left(\bar{\kappa} / \omega_{0}\right)^{2} n_{\gamma}(t)$ during the DCE. This atomic excitation is usually smaller than $N_{\text {Ryd }} \sim 100-$ 1000 , for example, for $\bar{\kappa} / \omega_{0} \sim 10^{-5}$ and $n_{\gamma}<10^{10}$. Anyway, the created photons are detected with the atoms efficiently after the DCE.

\section{EXPERIMENTAL REALIZATION}

We now discuss a feasible experimental realization of DCE with a semiconductor plasma mirror $[15,23]$. Based on the analyses presented so far for the DCE and photon detection, we can find desired values for the physical parameters.

The photons are created as

$$
n_{\gamma} \sim \frac{1}{4} e^{2 \chi t_{1}} \sim \frac{1}{4} e^{2 \pi\left(\chi / \omega_{0}\right) N_{\text {pulse }}}
$$

with the squeezing rate $\chi$ for the resonant mode, where $t_{1}=$ $N_{\text {pulse }}(2 \pi / \Omega)$ and $\Omega \simeq 2 \omega_{0}$ (see also Fig. 2 ). Hence the desired number $n_{\gamma}$ of created photons places a requirement for the squeezing rate as

$$
\chi / \omega_{0} \sim \frac{\ln \left(4 n_{\gamma}\right)}{2 \pi N_{\text {pulse }}} .
$$

Typically, $\chi \sim 0.01 \omega_{0}$ to obtain $n_{\gamma} \sim 10^{6}-10^{8}$ with $N_{\text {pulse }}=$ 300 laser pulses, where the threshold condition $\chi>\Gamma / 2$ for the DCE is also satisfied sufficiently with $Q \gtrsim 10^{3}$.

The effective displacement in Eq. (21) is achieved by applying a laser power $W_{\text {laser }} /$ pulse for the period $T=$ $2 \pi / \Omega \sim 0.2 \mathrm{~ns}$ :

$$
\delta_{m} / L \sim\left(n_{\mathrm{s}} e^{2} / \epsilon_{0} m_{*}\right) L / \pi^{2},
$$

where $\sin ^{2} k l=1$ for definiteness (the slab is placed in the middle of cavity $l=L / 2), \omega_{0} L \sim \pi$, and $n_{\mathrm{s}}=n_{e} \delta\left(\propto W_{\text {laser }}\right)$ is the surface number density of electrons. We may readily obtain $\left(n_{\mathrm{s}} e^{2} / \epsilon_{0} m_{*}\right) L \sim 1$ with a reasonable laser power $W_{\text {laser }} /$ pulse $\sim 0.01 \mu \mathrm{J} /$ pulse [23], achieving a significant displacement $\delta_{m} \sim 0.1 L$. In this case, the conductivity effect $\delta_{m}$ in Eq. (21) dominates the dielectric effect $\delta_{\epsilon}$ in Eq. (20) for $\epsilon_{1}(0) \sim 1-10$ and $\epsilon_{1}(0) \leqslant\left|\epsilon_{1}(t)\right|$ [the photon damping by the complex $\epsilon_{1}(t)$ does not exceed the squeezing by the DCE mainly with $\delta_{m}$ ]. We estimate the variation of the mode frequency as

$$
\delta \omega \simeq\left(\delta_{m} / L\right) \omega_{0} \sim 0.1 \omega_{0}\left(W_{\text {laser }} / 0.01 \mu \mathrm{J}\right) .
$$


By noting the relation $|\delta \omega| \simeq|2 g|$, the desired squeezing rate for the DCE can be obtained in Eq. (33) with $\Delta=0$ as

$$
\chi=\left|2\langle g\rangle_{\Omega}\right| \sim 0.01 \omega_{0}\left(r_{\Omega} / 0.1\right)\left(W_{\text {laser }} / 0.01 \mu \mathrm{J}\right) .
$$

Here the factor $r_{\Omega}$ represents the Fourier component $\langle g\rangle_{\Omega} e^{-i \Omega t}$ of $g(t)$, which may be optimized by suitably designing the time profile $W_{\text {laser }}(t)$ of the laser pulse. As seen in Eqs. (34) and (35), the tuning of $\Omega$ is required for the resonance by taking into account the average shift $\langle\delta \omega\rangle / \omega_{0} \sim 0.01-0.1$.

As for the photon detection, the analyses in Sec. V indicate that roughly $N_{\mathrm{Ryd}} / 2 \sim 100$ atomic excitations are detected per mean atomic transit time $t_{\text {tr }} \sim 0.1 \mathrm{~ms}$ for the creation of $n_{\gamma} \sim 10^{6}-10^{8}$ photons via the DCE. The quality factor of the cavity should be chosen suitably to ensure the efficient atomic excitation and detection. Specifically, $Q \sim 5 \times 10^{3}$ in Eq. (63) or $Q \gtrsim 5 \times 10^{6}$ in Eq. (65). We note that even if an excessive number of photons $\left(n_{\gamma} \gg 10^{8}\right)$ are created, their detection is actually limited by the number of Rydberg atoms $N_{\text {Ryd }} \sim 100-1000$. After the detection, the photons remaining in the cavity are relaxed, finally, as $n_{\gamma} \rightarrow 0$ for $t \gtrsim 10 \mathrm{~ms} \gg \Gamma^{-1}, t_{\mathrm{tr}}$; namely, the field returns to the vacuum. Then the subsequent rounds of photon creation and detection are performed repeatedly.

\section{SUMMARY}

We have investigated quantum mechanically the photon creation via DCE and its detection with Rydberg atoms, specifically considering the experimental realization in a resonant cavity with a plasma mirror of a semiconductor slab irradiated by laser pulses. The canonical Hamiltonian for the DCE is derived in terms of the creation and annihilation operators, showing the explicit time variation which originates from the external configuration such as the nonstationary plasma mirror. Then the photon creation is evaluated as squeezing from the Heisenberg equations. This confirms that a sufficiently large number of photons can be created via the DCE with a reasonable squeezing rate when the laser pulses are applied many times. The atomic excitation process to detect the photons is described with the atom-field interaction, which clarifies the conditions for the efficient detection. Based on these analyses, desired values of the physical parameters are considered for a feasible experiment for DCE and its detection with a plasma mirror and Rydberg atoms.

\section{ACKNOWLEDGMENTS}

The authors appreciate valuable discussions from $\mathrm{S}$. Matsuki, Y. Kido, T. Nishimura, W. Naylor, and the Ritsumeikan University group.
[1] H. B. G. Casimir, Proceedings of the Royal Netherlands Academy of Arts and Sciences 51, 793 (1948).

[2] L. Parker, Phys. Rev. Lett. 21, 562 (1968); Phys. Rev. 183, 1057 (1969).

[3] G. T. Moore, J. Math. Phys. 11, 2679 (1970).

[4] S. A. Fulling and P. C. W. Davies, Proc. R. Soc. London, Sect. A 348, 393 (1976).

[5] M. Razavy and J. Terning, Phys. Rev. D 31, 307 (1985).

[6] H. Johnston and S. Sarkar, J. Phys. A 29, 1741 (1996).

[7] G. Barton and C. Eberlein, Ann. Phys. 227, 222 (1993); G. Barton and A. Calogeracos, ibid. 238, 227 (1995).

[8] A. Lambrecht, M.-T. Jaekel, and S. Reynaud, Phys. Rev. Lett. 77, 615 (1996).

[9] C. K. Law, Phys. Rev. A 49, 433 (1994).

[10] R. Schützhold, G. Plunien, and G. Soff, Phys. Rev. A 57, 2311 (1998).

[11] V. V. Dodonov, Phys. Lett. A 207, 126 (1995); V. V. Dodonov and A. B. Klimov, Phys. Rev. A 53, 2664 (1996).

[12] V. V. Dodonov, Phys. Rev. A 58, 4147 (1998); Phys. Lett. A 244, 517 (1998).

[13] M. Crocce, D. A. R. Dalvit, and F. D. Mazzitelli, Phys. Rev. A 64, 013808 (2001).
[14] H. Saito and H. Hyuga, Phys. Rev. A 65, 053804 (2002).

[15] C. Braggio et al., Rev. Sci. Instrum. 75, 4967 (2004); Europhys. Lett. 70, 754 (2005).

[16] E. Yablonovitch, Phys. Rev. Lett. 62, 1742 (1989).

[17] Yu. E. Lozovik, V. G. Tsvetus, and E. A. Vinogradov, Phys. Scr. 52, 184 (1995); JETP Lett. 61, 723 (1995) [Pis'ma Zh. Eksp. Teor. Fiz. 61, 711 (1995)].

[18] M. Crocce, D. A. R. Dalvit, F. C. Lombardo, and F. D. Mazzitelli, Phys. Rev. A 70, 033811 (2004).

[19] M. Uhlmann, G. Plunien, R. Schützhold, and G. Soff, Phys. Rev. Lett. 93, 193601 (2004).

[20] A. V. Dodonov and V. V. Dodonov, J. Opt. B 7, S47 (2005); V. V. Dodonov and A. V. Dodonov, J. Phys. A 39, 6271 (2006); J. Phys. B 39, S749 (2006).

[21] M. Ruser, J. Phys. A 39, 6711 (2006); Phys. Rev. A 73, 043811 (2006).

[22] J. Haro and E. Elizalde, Phys. Rev. Lett. 97, 130401 (2006); Phys. Rev. D 76, 065001 (2007).

[23] W. Naylor, S. Matsuki, T. Nishimura, and Y. Kido, Phys. Rev. A 80, 043835 (2009).

[24] M. Tada et al., Phys. Lett. A 349, 488 (2006).

[25] See, e.g., S. Haroche and J. Raimond, Adv. At. Mol. Phys. 20, 347 (1984). 